\title{
Wearable breathing barriers (masks) to reduce droplet transmission of COVID-19
}

Jeffrey L. Pellegrino, Ph.D. MPH

\begin{abstract}
People trained in first aid, may be called upon to provide immediate and long-term care because of their competencies, especially as traditional healthcare workers may be limited or not available. Providing care for a person with Covid-19 increases the risk to the person for disease transmission and requires a breathing barrier with a filter that is better than the common procedural mask or cloth mask. First aid educators are in a unique position within the community and organized health care to provide support for the development of breathing barrier construction that could be used by first aiders or other healthcare workers ( $\mathrm{HCW}$ ) when commercially made breathing respirators or filters are not available. This review and recommendations provides rationale and teaching elements for building the highest quality mask with given resources.
\end{abstract}

As part of crisis response, a clinical research inquiry into improvised and constructed masks to reduce droplet transmission of Covid-19 for persons caring in high risk situations took place March 24-28, 2020. Assumptions taken into consideration:

Crisis Capacity or no availability of disposable N95 filtering facepiece respirators (N95), as described by U.S. Centers for Disease Control (CDC) has been or will be reached by a facility, region, or larger entity. (Strategies for Optimizing the Supply of Facemasks: COVID-19 | CDC, n.d.; Strategies for Optimizing the Supply of N95 Respirators: COVID-19 | CDC, n.d.)

$>$ Increased vigilance to disinfecting surfaces and hand hygiene will be enforced

$>$ Eye-protection and other Personal Protective Equipment (PPE) are in continued use.

\section{Background}

The World Health Organization (WHO) recommends contact and droplet precautions and not routine use of airborne precautions for HCW providing care to patients with suspected/confirmed Covid-19, based on its MERS guidance, China's experience with COVID-19, and previous respiratory disease outbreaks of SARS and MERS (Infection prevention and control during health care when novel coronavirus $(n \mathrm{CoV})$ infection is suspected, n.d.). The CDC also comments on the mode of transmission is primarily via respiratory droplets, when the infected person coughs or sneezes (Infection Control: Severe acute respiratory syndrome coronavirus 2 (SARSCoV-2) | CDC, n.d.). Value of N95 masks to clinically prevent infection is not established (Cowling et al., 2010; Long et al., 2020; Smith et al., 2016). Aerosolized virus particles (20-100nm) are not filtered through medical/surgical masks in general (Bałazy et al., 2006; Lee et al., 2008), but may slow dispersion of droplets (Milton et al., 2013). This counters pre-Covid-19 pandemic best practices/ regulated practices of HCWs. Situational demands on resources will necessarily alter previous practices, which will require psychological, social, and practical behaviors.

PPE rationing starts with protecting the most vulnerable/ at risk. (Healthcare Supply of Personal 
Protective Equipment | CDC, n.d.). N95s provide some level of protection for high risk HCWs (Wang et al., 2020). Risk can be based on personal factors (age, chronic conditions, immunecompromise, etc.) or specific procedures (surgery, aerosol-generating procedures, patients with other infections- tuberculosis, measles, varicella, etc.) being done. Other engineering or physical controls should be exhausted prior to putting someone at increased risk. Physical distancing may be the easiest (Lai et al., 2012; Strategies for Optimizing the Supply of N95 Respirators: COVID-19 | $C D C$, n.d.).

A last option for when supplies of commercial N95 or medical masks are exhausted is the use of improvised breathing barriers. Constructed cloth breathing barriers - aka masks - have emerged in many forms by a concerned public. These are generally constructed to look like medical masks (aka procedural, or ones that loop over ears) by following a variety of internet directions. Only one academic article was found on creation and testing of a cloth breathing barrier (Dato et al., 2006). Other articles were identified in terms of filtration of materials. Fabric type matters to a certain extent.

The US Food and Drug Administration (FDA) standards for surgical masks (FAQs on Shortages of Surgical Masks and Gowns | FD $A$, n.d.) include fluid resistance, filtration efficiency, air exchange, flammability, and biocompatibility as criteria for commercial mask construction. Using these headings, I provide suggestions based on materials that may be found in a home to construct masks to the best available standard:

\section{$>$ Fluid resistance:}

- Outer material ideally would have some level of water resistance, the use of surgical drapery sheets has been suggested. Other options may include Gore-Tex or breathable fabric with water resistance.

Filtration efficiency:
- The size of particle ideally filtered is $<.125$ microns, however this ideal cannot be reached with homemade cloth masks. The more realistic size would be $>.3$ microns, assuming the virus is suspended in droplets.

- Davies (2013) identified vacuum cleaner bags as the best filtration material found in a home setting. Depending on type of bag it can filter down to .3 microns (How a Vacuum Bag Works - gkvacbags, n.d.). A double layered dish towel (aka tea towel) followed in efficacy for 1 micron particles (Davies et al., 2013; van der Sande et al., 2008). Hanes (70\% Cotton/ 30\% polyester) sweatshirt had better value than t-shirts, scarfs, or masks made for pollution in limiting penetration of .2 microns (Rengasamy et al., 2010). Dato (2006) used 10 layers of Hanes Heavyweight 100\% preshrunk cotton T-shirt (made in Honduras), boiled and dried to promote shrinking to achieve a filter.

$>$ Air exchange:

- A measure of breathability and comfort of masks is needed to maintain compliance of placement and length of use.

- Dato's (2006) prototype design was tested for fit for over an hour, without difficulty by authors of the manuscript and this author.

$>$ Flammability:

- Not considered because use of masks is in a healthcare setting or home.

> Biocompatibility

- Reducing the amount of contamination at any phase of use of the constructed mask is important. Dato (2006) t-shirts were sterilized by being boiled for 10 minutes, which also maximized shrinkage, prior to construction

- A sanitation process needs to be instituted, which could include

- 3-hour limit on wearing a single mask

- Durable fabric that can withstand decontamination (i.e., hot water, bleach) 
- Discardable filters (ex. vacuum cleaner bags pieces, multiple layers of recommended fabric)

\section{Practical Recommendation}

The following description is an enhanced prototype of the Dato (2006) design, which is the only one fit tested. It is modified with a nose forming pipe cleaner/ wire and a pocket that vacuum cleaner bag (Davies et al., 2013) filter pieces can be replaced. Alternatively, could be filled with 8 layers of heavy t-shirt material, as originally described by Dato (2006).

\section{Benefits:}

$>$ The outer mask can be washed/sterilized.

$>$ The increased coverage over a plain cloth with ear loops

$>$ This design also has a darted face piece to allow for easier breathing.

$>$ If materials were assembled, takes about 5 min to create with a few practices.

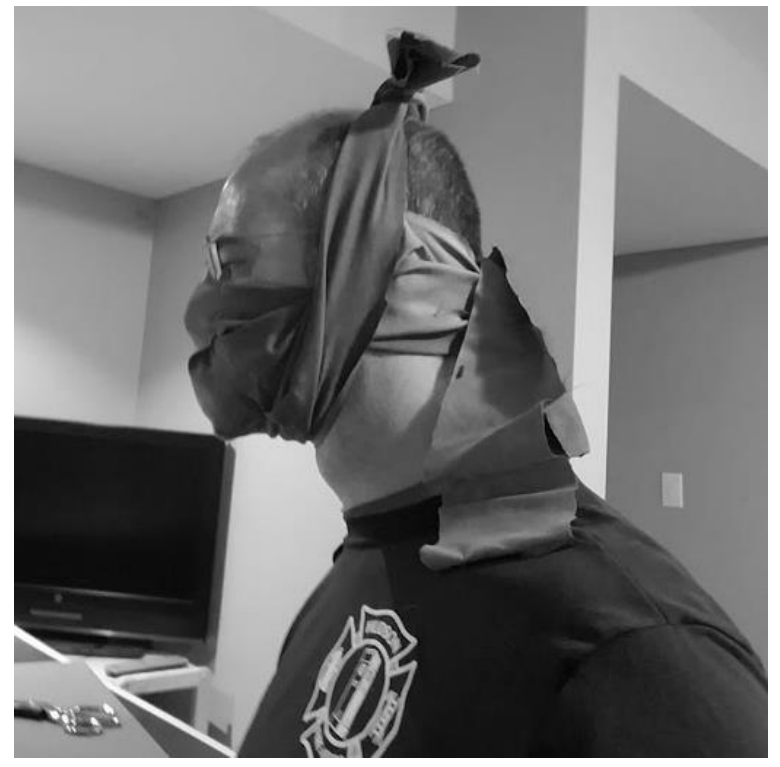

Materials \& tools:

- Scissors

- Marker

- Ruler

- Thread and needle
- Breathing cup template

- Outer fabric: $\approx 15$ ”h x $28 " w(\approx 37 \times 72$ $\mathrm{cm}$ ), best outer material available, see above recommendations

- Filter material: 6.5" x 5.5"

- Pocket material: 6" x 6", comfortable fabric for face contact

- Pipe cleaner or semi-flexible wire

Building:

1. Mark the outer fabric, fluid resistant side down, with a center line, to align filter pocket and filter material.

2. Trace filter pattern, approximately 3" from the bottom, and on the center line.

3. Mark darting locations.

4. Trace, cut, and mark darting locations on filter material and pocket material with breathing cup template.

5. Dart all markings, on the outer fabric, pocket fabric, and filter material, by folding the mark closest to the horizonal center to the corresponding mark (toward top or bottom) and stich the fold that was created to create a "cup".

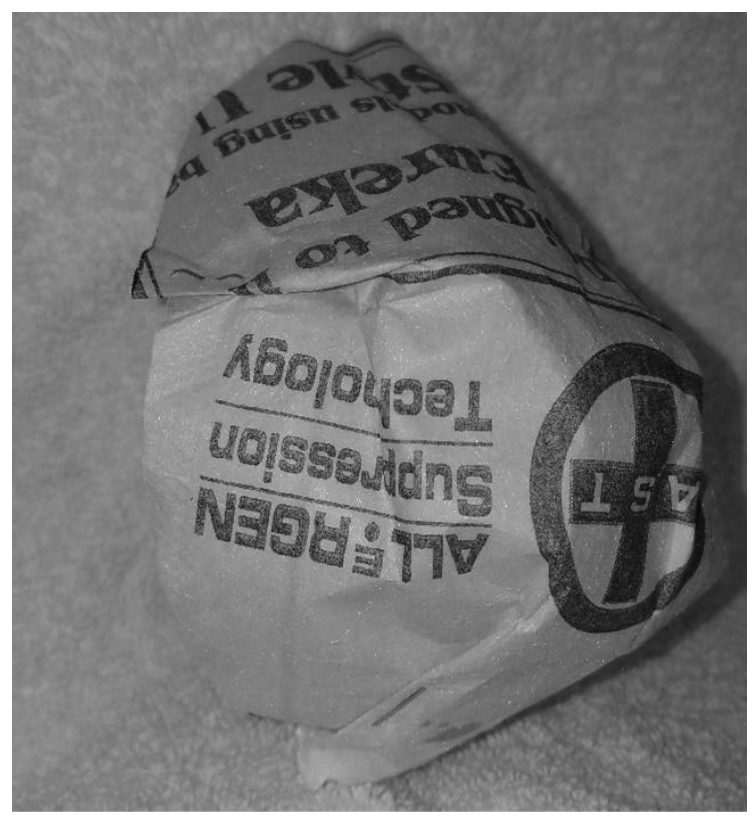

6. Stich the pocket on to the outer material, right, left, \& bottom, so the cups align. 
7. Measuring from the bottom of the outer fabric, mark a horizontal line $6 "(15 \mathrm{~cm})$ and 9" $(23 \mathrm{~cm})$. Cut from both ends to create ties.

Mask can be washed/ sterilized. For use, insert filter pieces* into pocket.

Putting on the mask (donning):

1. Insert filter $\operatorname{cup}(\mathrm{s})$ into pocket.

2. Place a thick pipe cleaner/ wire horizontally at the top of the mask, roll fabric toward filter pocket, this becomes Tie A.

3. Secure Tie A behind head (occipitally) with an overhand knot with an extra twist (Surgeon's knot) then into a bow. Adjust pipe cleaner/ wire to contour nose.

4. Tie $\mathrm{B}$, the bottom strip, is brought up over the top of the head and secured the same way.
5. Tie $\mathrm{C}$, the middle strip then is tied behind the head to help reduce gaps.

Removing the mask (doffing):

1. Start with good hand hygiene, release bows, without touching outside of mask

2. Remove filters, place mask in "dirty" bin or brown paper bag before cleaning.

3. Wash hands, then wash face.

Decontaminate mask with appropriate heat, chemical, or light before next use. Home cleaning includes washing with the warmest water possible and detergent. A bleach solution during the wash cycle is appropriate. Dry thoroughly. Dispose of dirty bag.

*Depending on filter material more than one layer may be desired.

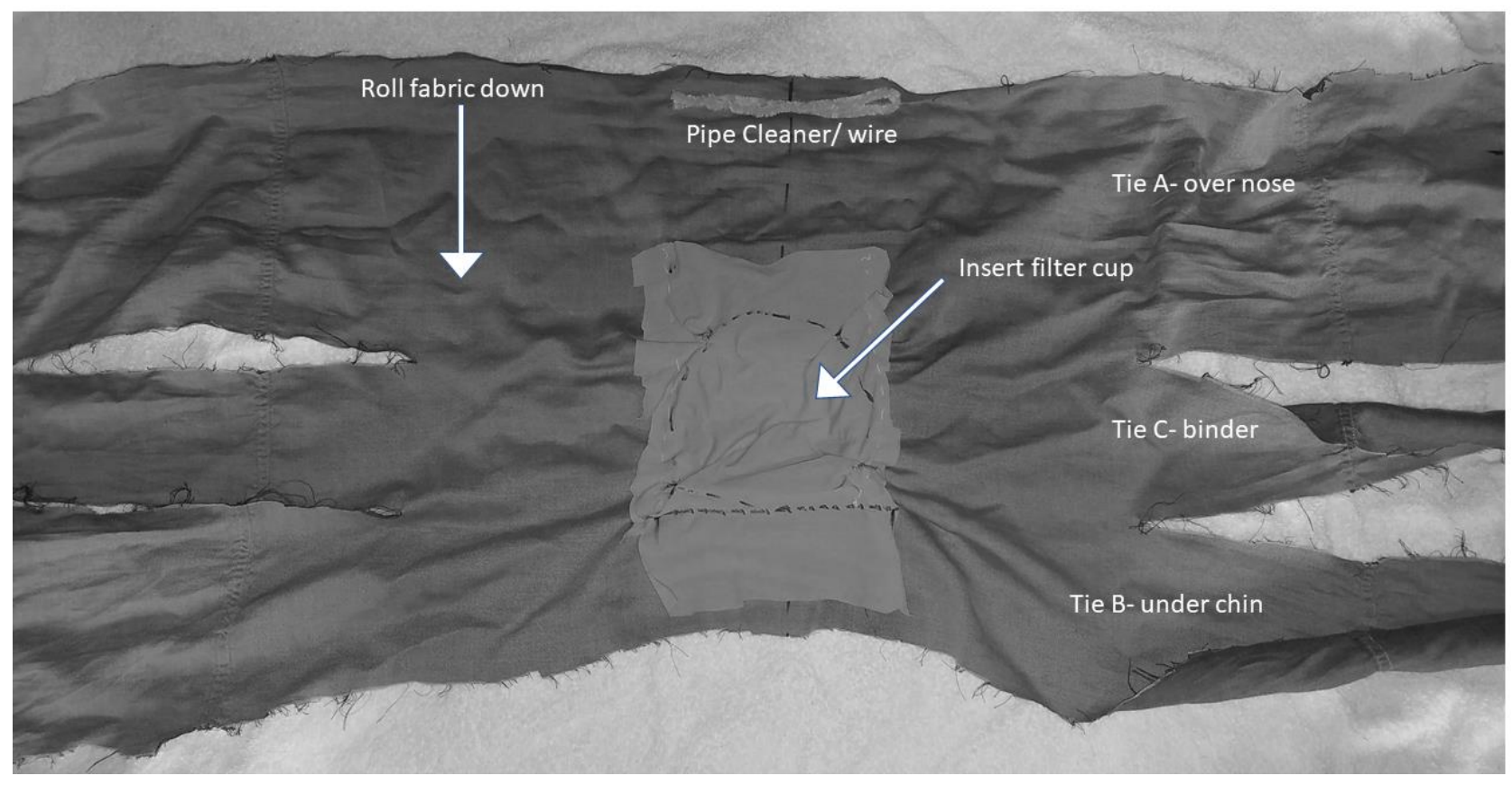

Figure 1: Prototype based on Dato (2006).

Insert filter cup. Place pipe cleaner/ wire at top of outer fabric. Roll fabric down to top of filter cup, which creates Tie A. Secure Tie A behind head. Secure Tie B under chin to top of head. Secure Tie C to bind mask behind head. 


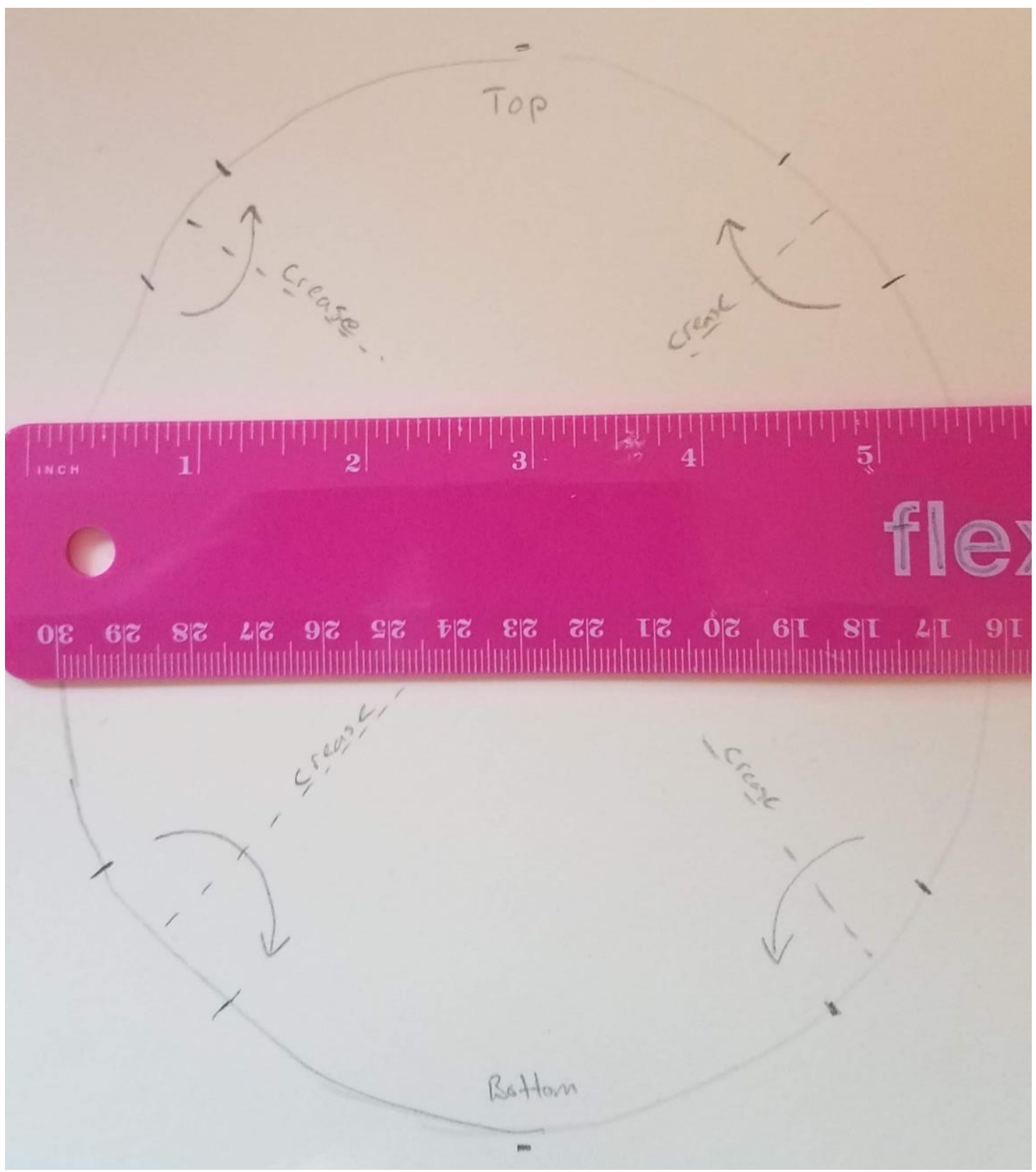

Figure 2: Template for breathing cup. Consider printing scaling by using the original scale. 


\section{References}

Bałazy, A., Toivola, M., Adhikari, A., Sivasubramani, S. K., Reponen, T., \& Grinshpun, S. A. (2006). Do N95 respirators provide $95 \%$ protection level against airborne viruses, and how adequate are surgical masks? American Journal of Infection Control, 34(2), 51-57. https://doi.org/10.1016/j.ajic.2005.08.018

Cowling, B. J., Zhou, Y., Ip, D. K. M., Leung, G. M., \& El, D. A. E. A. I. (2010). Face masks to prevent transmission of influenza virus: a systematic review. Epidemiological \& Infection, 138(4), 449-456. https://doi.org/10.1017/S0950268809991658

Dato, V. M., Hostler, D., \& Hahn, M. E. (2006). Simple respiratory mask. Emerging Infectious Diseases, 12(6), 1033-1034. https://doi.org/10.3201/eid1206.051468

Davies, A., Thompson, K.-A., Giri, K., Kafatos, G., Walker, J., \& Bennett, A. (2013). Testing the Efficacy of Homemade Masks: Would They Protect in an Influenza Pandemic? Disaster Medicine and Public Health Preparedness, 7(4), 413-418. https://doi.org/DOI: 10.1017/dmp.2013.43

FAQs on Shortages of Surgical Masks and Gowns / FDA. (n.d.). Retrieved March 24, 2020, from https://www.fda.gov/medical-devices/personal-protective-equipment-infection-control/faqsshortages-surgical-masks-and-gowns

Healthcare Supply of Personal Protective Equipment / CDC. (n.d.). Retrieved March 24, 2020, from https://www.cdc.gov/coronavirus/2019-ncov/hcp/healthcare-supply-ppe.html

How a Vacuum Bag Works - gkvacbags. (n.d.). Retrieved March 28, 2020, from https://gogreenklean.com/how-a-vacuum-bag-works/ 
Infection Control: Severe acute respiratory syndrome coronavirus 2 (SARS-CoV-2) / CDC. (n.d.). Retrieved March 24, 2020, from https://www.cdc.gov/coronavirus/2019-ncov/infection-control/controlrecommendations.html

Infection prevention and control during health care when novel coronavirus (nCoV) infection is suspected. (n.d.). Retrieved March 24, 2020, from https://www.who.int/publications-detail/infectionprevention-and-control-during-health-care-when-novel-coronavirus-(ncov)-infection-is-suspected20200125

Lai, A. C. K., Poon, C. K. M., \& Cheung, A. C. T. (2012). Effectiveness of facemasks to reduce exposure hazards for airborne infections among general populations. Journal of the Royal Society, Interface, 9(70), 938-948. https://doi.org/10.1098/rsif.2011.0537

Lee, S.-A., Grinshpun, S. A., \& Reponen, T. (2008). Respiratory Performance Offered by N95 Respirators and Surgical Masks: Human Subject Evaluation with $\mathrm{NaCl}$ Aerosol Representing Bacterial and Viral Particle Size Range. The Annals of Occupational Hygiene, 52(3), 177-185. https://doi.org/10.1093/annhyg/men005

Long, Y., Hu, T., Liu, L., Chen, R., Guo, Q., Yang, L., Cheng, Y., Huang, J., \& Du, L. (2020). Effectiveness of N95 respirators versus surgical masks against influenza: A systematic review and meta-analysis. Journal of Evidence-Based Medicine. https://doi.org/10.1111/jebm.12381

Milton, D. K., Fabian, M. P., Cowling, B. J., Grantham, M. L., \& McDevitt, J. J. (2013). Influenza virus aerosols in human exhaled breath: particle size, culturability, and effect of surgical masks. PLoS Pathogens, 9(3), e1003205-e1003205. https://doi.org/10.1371/journal.ppat.1003205

Rengasamy, S., Eimer, B., \& Shaffer, R. E. (2010). Simple Respiratory Protection-Evaluation of the Filtration Performance of Cloth Masks and Common Fabric Materials Against 20-1000 nm Size 
Practice consideration

Particles. Ann. Occup. Hyg, 54(7), 789-798. https://doi.org/10.1093/annhyg/meq044

Smith, J. D., MacDougall, C. C., Johnstone, J., Copes, R. A., Schwartz, B., \& Garber, G. E. (2016).

Effectiveness of $\mathrm{N} 95$ respirators versus surgical masks in protecting health care workers from acute respiratory infection: a systematic review and meta-analysis. CMAJ : Canadian Medical Association Journal = Journal de l'Association Medicale Canadienne, 188(8), 567-574.

https://doi.org/10.1503/cmaj.150835

Strategies for Optimizing the Supply of Facemasks: COVID-19 / CDC. (n.d.). Retrieved March 24, 2020, from https://www.cdc.gov/coronavirus/2019-ncov/hcp/ppe-strategy/face-masks.html

Strategies for Optimizing the Supply of N95 Respirators: COVID-19 / CDC. (n.d.). Retrieved March 24, 2020, from https://www.cdc.gov/coronavirus/2019-ncov/hcp/respirators-strategy/crisis-alternatestrategies.html

van der Sande, M., Teunis, P., \& Sabel, R. (2008). Professional and home-made face masks reduce exposure to respiratory infections among the general population. PloS One, 3(7), e2618-e2618. https://doi.org/10.1371/journal.pone.0002618

Wang, X., Pan, Z., \& Cheng, Z. (2020). Association between 2019-nCoV transmission and N95 respirator use. Journal of Hospital Infection. https://doi.org/10.1016/j.jhin.2020.02.021 